20

\title{
Диагностика диабета на основе анализа выдыхаемого воздуха методом терагерцовой спектроскопии и машинного обучения
}

\author{
() Ю.В. Кистенев ${ }^{1,2}$, А.В. Тетенева ${ }^{2}$, Т.В. Сорокина ${ }^{2}$, А.И. Князькова ${ }^{1,3}$, О.А. Захарова ${ }^{1,3}$, \\ А. Кюссе ${ }^{4}$, В.Л. Вакс ${ }^{5}$, Е.Г. Домрачева ${ }^{5}$, М.Б. Черняева ${ }^{5}$, В.А. Анфертьев ${ }^{5}$, Е.С. Сим ${ }^{1,2}$, \\ И.Ю. Янина ${ }^{1,6}$, В.В. Тучин ${ }^{1,6,7}$, А.В. Борисов ${ }^{1,2}$ \\ ${ }^{1}$ Национальный исследовательский Томский государственный университет, \\ 634050 Томск, Россия \\ ${ }^{2}$ Сибирский государственный медицинский университет, \\ 634050 Томск, Россия \\ ${ }^{3}$ Институт фризики прочности и материаловедения Сибирского отделения РАН, \\ 634055 Томск, Россия \\ ${ }^{4}$ Université du Littoral-Côte d'Opale, \\ 59140 Dunkerque, France \\ ${ }^{5}$ Институт физики микроструктур РАН, \\ 603087 Нижний Новгород, Россия \\ ${ }^{6}$ Саратовский национальный исследовательский государственный университет им. Н.Г. Чернышевского, \\ 410012 Саратов, Россия \\ ${ }^{7}$ Университет ИТМО, \\ 197101 Санкт-Петербург, Россия \\ e-mail: yuk@iao.ru
}

Поступила в редакцию 10.12.2019 г.

В окончательной редакции 07.02.2020 г.

Принята к публикации 28.02.2020 г.

\begin{abstract}
Представлены результаты исследования выдыхаемого воздуха пациентов с сахарным диабетом в сравнении со здоровыми добровольцами с использованием широкополосной ТГц спектроскопии во временной области. Выявлены характерные спектральные поддиапазоны, в которых профили спектров поглощения проб выдыхаемого воздуха целевой и контрольной групп отличаются наиболее существенно: $0.560,0.738$, $0.970,1.070,1.140,1.180,1.400$ ТГц. С помощью метода главных компонент показано, что совокупность коэффициентов поглощения в этих областях позволяет надежно разделить целевую и контрольную группы. Проведено сравнение полученных результатов с измерениями паров ацетона в воздухе пациентов с сахарным диабетом и здоровых добровольцев.
\end{abstract}

Ключевые слова: диабет, выдыхаемый воздух, терагерцовая спектроскопия, машинное обучение.

DOI: $10.21883 /$ OS.2020.06.49414.46-20

\section{Введение}

Среди заболеваний, связанных с нарушением метаболизма, наиболее тяжелые социально-экономические потери общество несет от сахарного диабета (СД) и его осложнений. В 2016 г. 415 миллионов человек болели СД, прогноз на 2040 г. - 642 миллиона человек [1,2]. Официально в России зарегистрировано более 3 млн больных СД, однако по оценкам эпидемиологических исследований их реальное число около 9-10 миллионов человек [3]. Ежегодные прямые расходы на СД приближаются к 1 трлн долларов.

Диагностика СД осуществляется путем исследования клинико-лабораторных маркеров заболевания, включая контроль уровня глюкозы, гликированного гемоглобина, С-пептида, инсулина и др. Непрерывный мониторинг уровня глюкозы в крови также играет важную роль в оценке эффективности и безопасности лечения в некоторых подгруппах пациентов с сахарным диабетом 1-го типа и у отдельных пациентов с сахарным диабетом 2-го типа [4]. Контроль за уровнем гликемии требуется и для динамического наблюдения за пациентами и коррекции их состояния. Все эти тесты инвазивны, требуют времени и расходных материалов. Поэтому методы быстрой неинвазивной диагностики СД и оценки состояния таких пациентов представляют большой практический интерес.

Регистрация летучих молекул в выдыхаемом воздухе является перспективным подходом для оперативного неинвазивного анализа метаболических процессов в организме [5-7]. В работе [8] были проанализированы летучие органические вещества (ЛОВ) в выдыхаемом воздухе у пациентов с диабетом 1-го и 2-го типов и здоровых добровольцев. ЛОВ включали метилированные алканы, $\mathrm{C}_{4}-\mathrm{C}_{20}$ алканы и монометилированные алканы. Показано, что при гипергликемии в выдыхаемом воздухе наблюдается повышенное содержание метилнитрата [9]. Ключевым фактором в развитии осложнений 


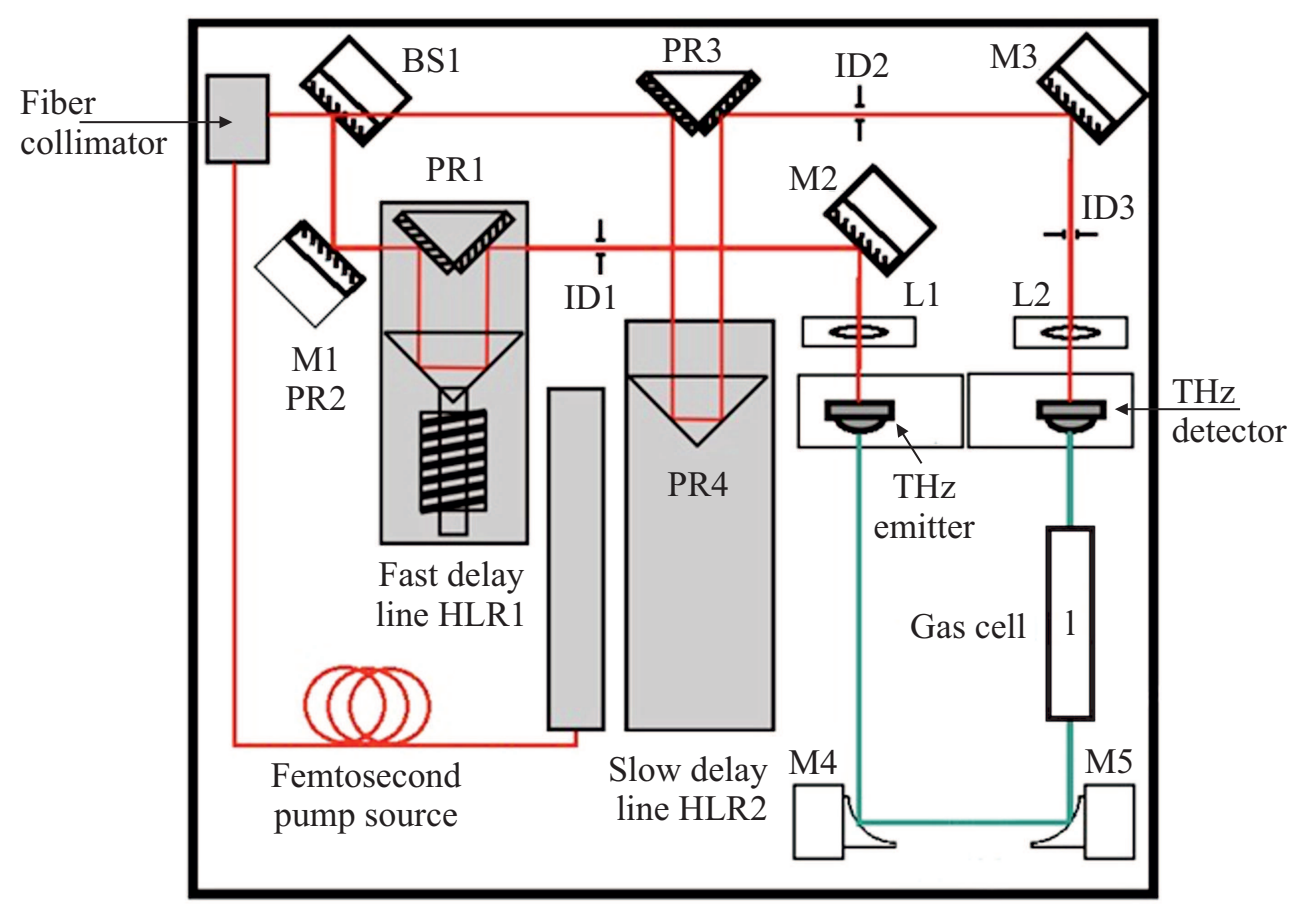

Рис. 1. Оптическая схема спектрометра „Т-Sрес“ с дополнительной газовой кюветой.

при СД является оксидативный стресс. Оксидативный стресс вовлечен в развитие основных осложнений СД, включая ретинопатию, нефропатию, невропатию и ускоренное развитие ишемической болезни сердца. Среди маркеров оксидативного стресса можно выделить перекисное окисление липидов и рост NO в выдыхаемом воздухе [10]. Большинство исследований по перекисному окислению липидов связано с исследованием малондиальдегида и подобных ему компонент. Продукты окисления белков более стабильны и легко контролируемы при оксидативном стрессе [11]. СД влияет на содержание СО, $\mathrm{CO}_{2}$, этанола, алканов [12].

Ацетон является одним из основных ЛОВ в организме человека [13]. Существует корреляция между содержанием ацетона в выдыхаемом воздухе и уровнем гипергликемии [14-18]. Контроль ацетона в выдыхаемом воздухе обеспечивает $76 \%$ чувствительности и $81 \%$ специфичности при разделении пациентов с диабетом и здоровых лиц, контроль 8 метаболитов, включая изопрен, бутанол, обеспечивает чувствительность 90\% и специфичность $92 \%$ [12]. Таким образом, для надежного обнаружения определенной стадии заболевания необходим контроль достаточно большого числа ЛОВ.

Среди многообразия аппаратных методов анализа выдыхаемого воздуха достаточно привлекательны по совокупности характеристик методы лазерной спектроскопии [18].

Исследования выдыхаемого воздуха методами лазерной спектроскопии проводились в основном в ИК диапазоне [6,18-23]. Область терагерцовых (THz) длин волн интересна тем, что здесь присутствуют вращательные переходы ряда легких молекул, представляющих диагностический интерес $[24,25]$. Для регистрации спектров поглощения газовых образцов могут применяться методы $\mathrm{THz}$ спектроскопии высокого разрешения [26]. Наряду с очевидными преимуществами они имеют небольшой диапазон сканирования, что ограничивает число одновременно детектируемых ЛОВ в выдыхаемом воздухе. Техника $\mathrm{THz}$ спектроскопии во временной области (time-domain, TD) не обладает высоким спектральным разрешением, но обеспечивает быструю регистрацию спектров поглощения газовых образцов в широком диапазоне частот (типичное значение $0.1-3.5 \mathrm{THz}$ ).

Цель данной работы заключается в сравнении двух методов $\mathrm{THz}$ спектроскопии, использующих сигналы во временной и спектральных областях, и оценке их чувствительности, необходимой для проведения диагностики и мониторинга состояния пациентов, больных сахарным диабетом, на основе спектрального анализа выдыхаемого воздуха.

\section{Материалы и методы}

В исследовании участвовало 11 пациентов с СД и 11 здоровых добровольцев. Забор проб выдыхаемого воздуха осуществлялся в специальный контейнер объемом 11. Начальная часть выдоха объемом около 0.51 выдыхалась в окружающее пространство, поскольку она не несет полезной информации. Перед измерениями состояние пациентов с СД контролировалось клиническими методами, включая контроль уровня гликирован- 


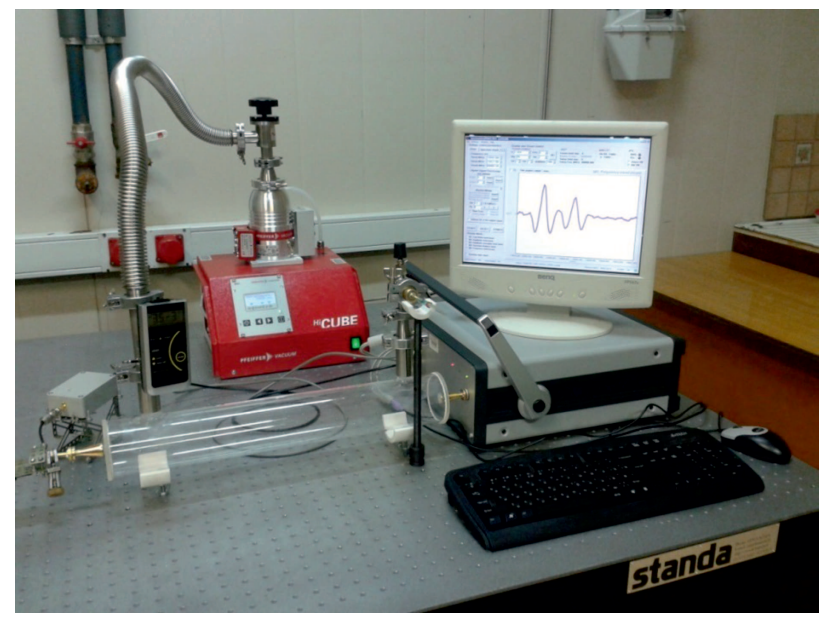

Pис. 2. Спектрометр $\mathrm{THz}$ диапазона с фазовой манипуляцией воздействующего излучения с источником излучения на основе лампы обратной волны.

ного гемоглобина и С-пептида, биохимический анализ крови, общий анализ крови, общий анализ мочи [27]. Для здоровых добровольцев предварительно измерялся уровень глюкозы глюкометром Accu-Chek Active. Условия измерения: утром до приема пищи.

Спектральное исследование выдыхаемого воздуха проводилось $\mathrm{THz}-\mathrm{TD}-$ спектрометром T-Spec фирмы EXPLA (рис. 1). Для этого использовалась дополнительная кювета с окнами из оптически прозрачного в $\mathrm{THz}$ области материала длиной $19.5 \mathrm{~cm}$, которая размещалась в оптическом тракте на пути $\mathrm{THz}$ волны (рис. 1). Продувка кюветы осуществлялась мембранным вакуумным насосом-компрессором NMP 05 S KNDCDC-5V фирмы CZL (расход 0.41/min) в течение $5 \mathrm{~min}$. Перед каждым измерением снимался референтный сигнал спектрометра с кюветой, заполненной воздухом.

Спектры интенсивности $\mathrm{THz}$ сигнала проб выдыхаемого воздуха $I_{\text {air }}$ и референтного сигнала $I_{\text {ref }}$ получали путем преобразования Фурье временной формы коротких импульсов, регистрируемых приемником [28]. Эти сигналы можно представить в виде

$$
\begin{gathered}
I_{\text {air }}=I_{0} \exp \left(-\left(k_{\text {air }} l_{1}+k_{\text {air }} l_{2}+k_{w} l_{3}\right)\right), \\
I_{\text {EAS }}=I_{0} \exp \left(-\left(k_{\mathrm{EAS}} l_{1}+k_{\text {air }} l_{2}+k_{w} l_{3}\right)\right) .
\end{gathered}
$$

Здесь $I_{0}$ - интенсивность сигнала, генерируемого $\mathrm{THz}$ эмиттером (рис. 1), $k_{\text {air }}-$ коэффициент поглощения атмосферного воздуха, $k_{w}-$ коэффициент поглощения окон кюветы, $k_{\mathrm{EAS}}-$ коэффициент поглощения пробы выдыхаемого воздуха в кювете. При этом коэффициент поглощения пробы в условных единицах можно представить в виде

$$
A=\left(k_{\mathrm{EAS}}-k_{\mathrm{air}}\right) l_{1}=-\log \left(\frac{I_{a} i r}{I_{\mathrm{ref}}}\right) .
$$

Сравнительный анализ профилей спектров поглощения выдыхаемого воздуха пациентов с СД и здоровых добровольцев в диапазоне $0.3-1.5 \mathrm{THz}$ осуществлялся с помощью метода главных компонент [19,29]. Достоинство метода заключается в возможности выделения наиболее информативных спектральных областей (информативные признаки), а также возможности визуализации взаимного расположения различных групп в пространстве признаков, которые связаны с информативными спектральными областями [30].

Дополнительно были проведены измерения содержания ацетона в образцах выдыхаемого воздуха в области 150.537 и $151.647 \mathrm{GHz}$ у условно здоровых добровольцев и пациентов с диабетом с использованием оригинального спектрометра высокого разрешения с
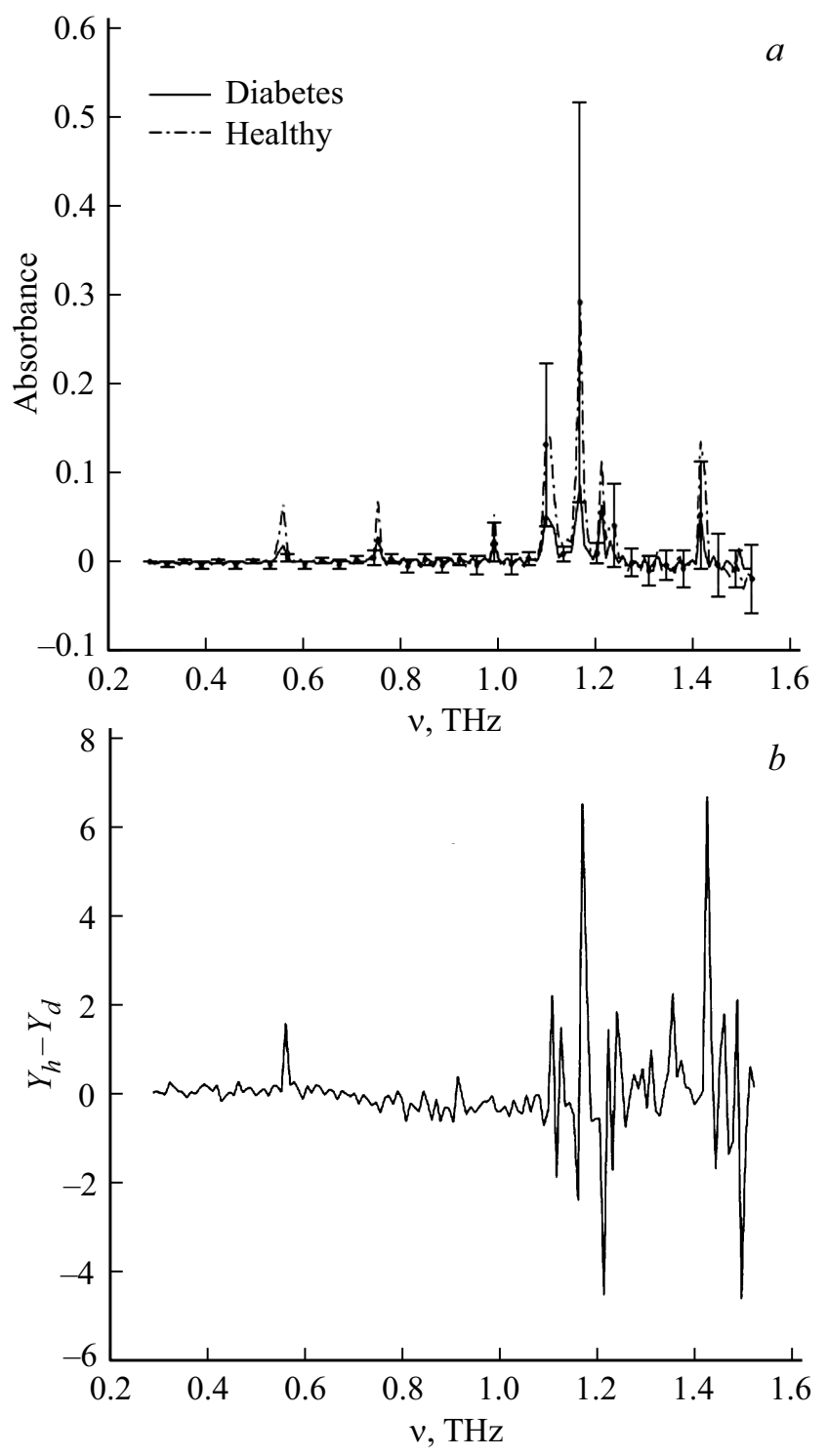

Рис. 3. Усредненные по группам спектры поглощения выдыхаемого воздуха $(a)$ и разность между средними значениями нормированных профилей спектров поглощения выдыхаемого воздуха контрольной и целевой групп $(b)$. 

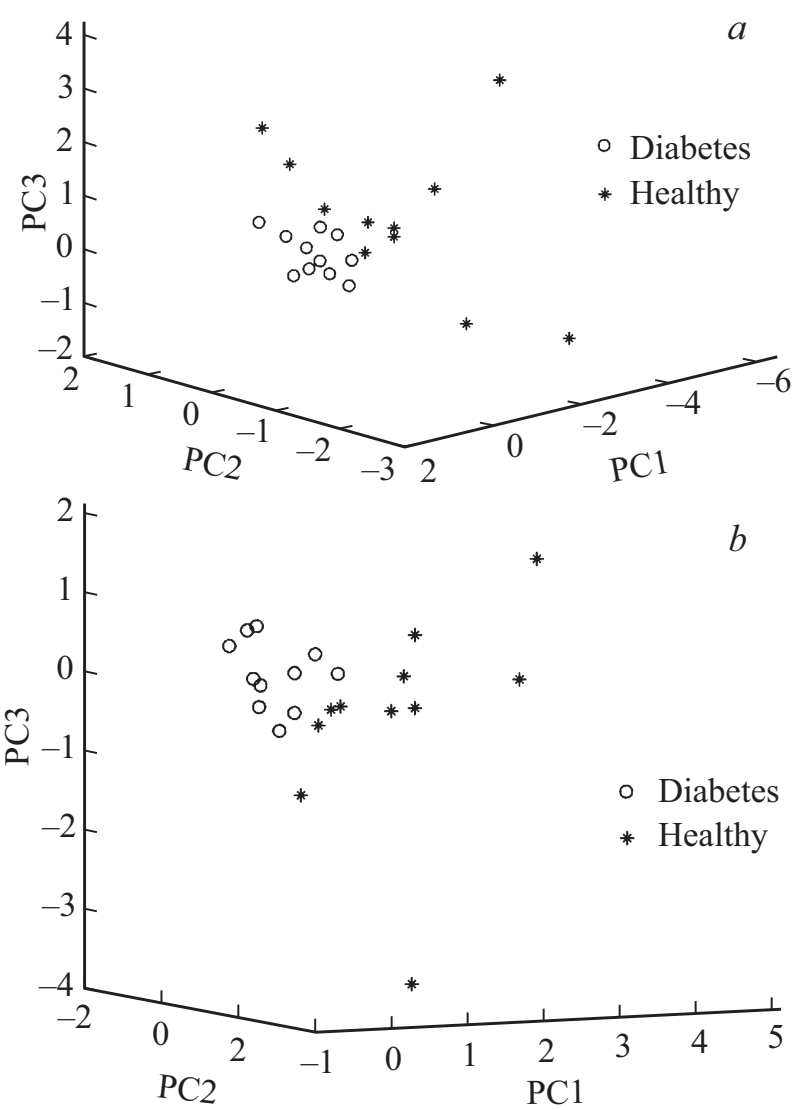

Рис. 4. Проекции профилей спектров поглощения выдыхаемого воздуха в широком спектральном диапазоне $(0.3-1.5 \mathrm{THz})$ исследуемых групп на подпространство 1, 2 и 3 главных компонент $(a)$, проекции узких спектральных подобластей с центрами, которые соответствовали характерным частотным областям (2), на подпространство 1, 2 и 3 главных компонент $(b)$.

фазовой манипуляцией воздействующего на газ излучения с источником излучения на основе лампы обратной волны (рис. 2) [31], работающего в диапазоне 118-178 GHz. Спектрометр основан на нестационарных эффектах, включая затухание свободной поляризации или быстрое свипирование частоты. Возникающие в результате этого переходные сигналы регистрируются в приемной части спектрометра. По величине и форме этих сигналов с высокой точностью и производится определение концентрации исследуемых компонент газовой смеси. В спектрометре используется система фазовой автоподстройки частоты (ФАПЧ) для автоматического контроля частоты лампы обратной волны и фазового сдвига. Длина измерительной кварцевой ячейки - $1 \mathrm{~m}$. Рабочее давление в ней поддерживается на уровне $(1-5) \cdot 10^{-2}$ mbar. Чувствительность спектрометра составляет порядка $5 \cdot 10^{-10} \mathrm{~cm}^{-1}$. Точность измерения интенсивности линии поглощения (без предварительной калибровки) не хуже 5\%. Точность установки частоты составляет не хуже $10^{-9}$ от несущей.

\section{Результаты}

В таблице представлены результаты измерения концентрации ацетона на линиях поглощения 150.537 и $151.647 \mathrm{GHz}$ в образцах воздуха, выдыхаемого условно здоровыми добровольцами и пациентами с диабетом. Видно, что перерыв в питании в течение ночи дает разнонаправленную динамику изменения уровня паров
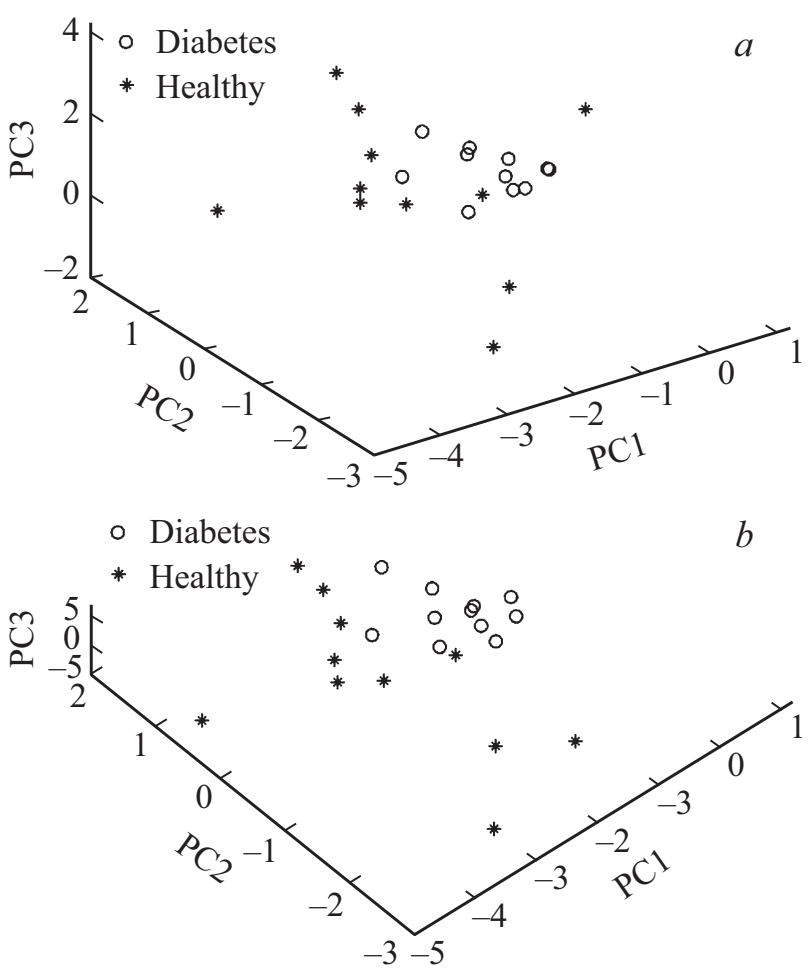

Рис. 5. Проекции профилей спектров поглощения выдыхаемого воздуха на подпространство 1, 2 и 3 главных компонент для поддиапазонов $0.52-0.60(a)$ и $1.36-1.44 \mathrm{THz}(b)$.

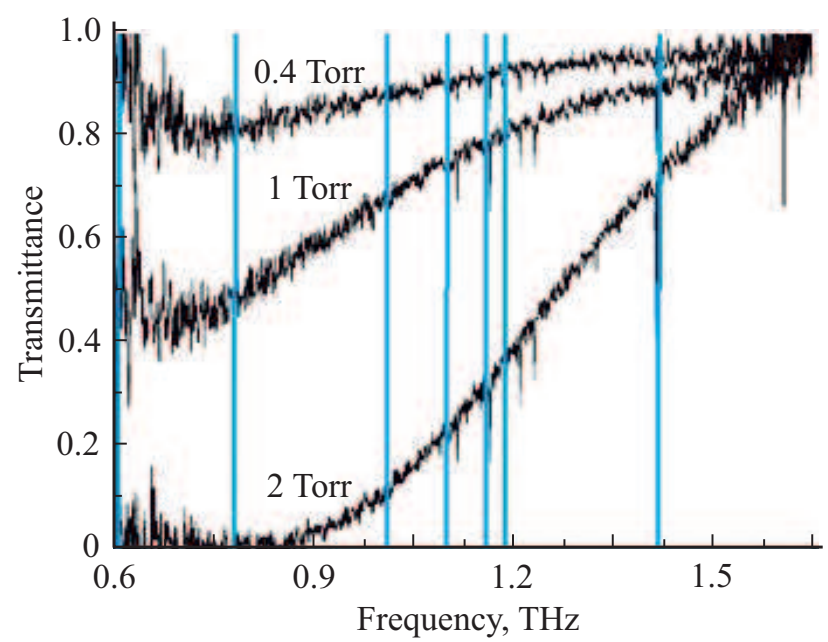

Рис. 6. Спектры пропускания паров ацетона при давлении в измерительной ячейке $0.4,1,2$ Torr. Вертикальные линии соответствуют характерным частотам в спектрах поглощения выдыхаемого воздуха (2). 
Содержание ацетона в образцах выдыхаемого воздуха

\begin{tabular}{c|c|c|c|c}
\hline $\begin{array}{c}\text { № } \\
\text { паци- } \\
\text { ента }\end{array}$ & $\begin{array}{c}\text { Условно } \\
\text { здоровые } \\
\text { добро- } \\
\text { вольцы, } \\
\text { рpm }\end{array}$ & $\begin{array}{c}\text { Условно } \\
\text { здоровые } \\
\text { добровольцы } \\
\text { натощак, } \\
\text { рpm }\end{array}$ & $\begin{array}{c}\text { Пациенты } \\
\text { д диабетом, } \\
\text { рpm }\end{array}$ & $\begin{array}{c}\text { Пациенты } \\
\text { диабетом, } \\
\text { натощак, } \\
\text { рpm }\end{array}$ \\
\hline 1 & 0.904 & 6.512 & 35.235 & 10.396 \\
2 & 1.909 & 9.349 & 24.654 & 9.334 \\
3 & 2.212 & 7.202 & 27.356 & 8.276 \\
4 & 1.413 & 7.672 & 15.657 & 8.571 \\
5 & 1.356 & 9.613 & 26.458 & 8.385 \\
6 & 1.172 & 13.405 & 32.651 & 8.384 \\
7 & 31.415 & 10.663 & & \\
8 & 29.782 & 9.99 & &
\end{tabular}

ацетона в выдыхаемом воздухе у здоровых добровольцев и пациентов с диабетом.

На рис. 3, а показаны усредненные спектры поглощения выдыхаемого воздуха для целевой и контрольной групп, полученные с использованием THz-TD-спектрометра в соответствии с формулой (1). Наиболее сильные отличия между группами наблюдаются в области частот 0.56, 0.74, 0.97, 1.07, 1.14, 1.18, $1.41 \mathrm{THz}$. (2)

Отметим, что практически все указанные области содержат линии поглощения водяного пара [32].

Для оценки возможности разделения групп на основе сравнения профилей спектров поглощения выдыхаемого воздуха в диапазоне $0.3-1.5 \mathrm{THz}$ был использован метод главных компонент (рис. 4,a). Видно хорошее разделение групп в пространстве главных компонент. Затем мы вырезали узкие спектральные подобласти (ширина каждой - $0.04 \mathrm{THz}$ ) с центрами, которые соответствовали характерным частотным областям (2), и затем использовали их совокупность в качестве характерных признаков групп. Представление этих признаков в пространстве главных компонент показано на рис. 4, $b$. Видно, что качество разделения групп в пространстве главных компонент остается достаточно высоким. Это свидетельствует о том, что данные характерные признаки являются определяющими для разделения групп.

На рис. 5 показаны проекции профилей спектров поглощения выдыхаемого воздуха для поддиапазонов $0.52-0.60$ и $1.37-1.45 \mathrm{THz}$ в отдельности на подпространство 1, 2 и 3 главных компонент. Видно, что использование отдельных поддиапазонов дает несколько худшее разделение групп в пространстве главных компонент. Данный результат, возможно, обусловлен более сильным влиянием других компонент, присутствующих в воздухе, в отдельном спектральном интервале.

На рис. 6 показаны спектры пропускания паров ацетона при различных давлениях, зарегистрированные с помощью фурье-спектрометра с разрешением $0.04 \mathrm{~cm}^{-1}$ [33]. Видно, что характерные для пациентов с СД спектральные признаки (2) частично совпадают с областями поглощения паров ацетона. Таким образом, увеличение содержания паров ацетона в выдыхаемом воздухе характерно для пациентов с СД, однако присутствуют и другие маркеры, представленные во введении, в частности $\mathrm{CO}, \mathrm{CO}_{2}$, этанол, алканы [12].

\section{Заключение}

В работе исследованы $\mathrm{THz}$ спектры поглощения выдыхаемого воздуха пациентов с СД и здоровых добровольцев. Выделены характерные спектральные области, в которых профили спектров поглощения проб выдыхаемого воздуха целевой и контрольной групп отличаются наиболее существенно: $0.560,0.738,0.970,1.070,1.140$, 1.180, 1.400 THz. С помощью метода главных компонент показано, что совокупность коэффициентов поглощения в этих областях позволяет надежно разделить целевую и контрольную группы.

Таким образом, THz-TD-спектроскопия является полезным инструментом для оценки возможности использования выдыхаемого воздуха как диагностического агента.

\section{Благодарности}

Работа выполнена в рамках Программы фундаментальных научных исследований государственных академий наук на 2013-2020 гг. направление III.23.

\section{Финансирование работы}

Исследование выполнено при финансовой поддержке РФФИ в рамках научных проектов № 18-52-16025, № 17-00-00275 (17-00-00272, 17-00-00184, 17-00-00186).

\section{Соблюдение этических стандартов}

Все процедуры, выполненные в исследовании с участием людей, соответствуют Хельсинкской декларации 1964 г. и ее последующим изменениям или сопоставимым нормам этики. От каждого из включенных в исследование участников было получено информированное добровольное согласие.

\section{Конфликт интересов}

Авторы заявляют, что у них нет конфликта интересов.

\section{Список литературы}

[1] Shestakova M.V., Galstyan G.R. // Problems of Endocrinology. 2017. V. 63. N 4. P. 257. doi 10.14341/probl2017634257-268

[2] Asfandiyarova N.S. // Diabetes Mellitus. 2015. V. 18. N 4. P. 12. doi 10.14341/DM6846

[3] Dedov I.I., Shestakova M.V., Galstyan G.G. // Diabetes Mellitus. 2016. V. 19. N 2. P. 104. doi 10.14341/DM2004116-17

[4] Singh M., Pal R., Ranjan R., Sarkar G., Bharti R., Pal S. // JKIMSU. 2017. V. 6. N 4. P. 12. 
[5] Boots A.W., Bos L.D., van der Schee M.P., van Schooten F.J, Sterk P.J. // Trends Mol. Med. 2015. V. 10. N 21. P. 633. doi 10.1016/j.molmed.2015.08.001

[6] Kistenev Y.V., Borisov A.V., Kuzmin D.A., Bulanova A.A. // AIP Conf. Proc. 2016. V. 1760. P. 020028. doi $10.1063 / 1.4960247$

[7] Kistenev Y.V., Borisov A.V., Kuzmin D.A., Penkova O.V., Kostyukova N.Y., Karapuzikov A.A. // JBO. 2017. V. 22. N 1. P. 017002. doi 10.1117/1.JBO.22.1.017002

[8] Phillips M., Cataneo R.N., Cheema T., Greenberg J. // Clin. Chim. Acta. 2004. V. 344. P. 189. doi 10.1016/j.ccen.2004.02.025

[9] Novak B.J., Blake D.R., Meinardi S., Rowland F.S., Pontello A., Cooper D.M., Galassetti P.R. // PNAS. 2007. V. 104. N 40. P. 15613. doi 10.1073/pnas.0706533104

[10] Stephens J.W., Khanolkar M.P., Bain S.C. // Atherosclerosis. 2009. V. 202. N 2. P. 321. doi 10.1016/j.atherosclerosis.2008.06.006

[11] Krzystek-Korpacka M., Salmonowicz B., Boehm D., Berdowska I., Zielinski B., Patryn E., Noczynska A., Gamian A. // Clinical Biochemistry. 2008. V. 41. P. 48. doi 10.1016/j.clinbiochem.2007.10.003

[12] Greiter M.B., Keck L., Siegmund T., Hoeschen C., Oeh U., Paretzke H.G. // Diabetes Technology \& Therapeutics. 2010. V. 12. N 6. P. 455.

[13] Das S., Pal S., Mitra M. // J. Med. Biol. Eng. 2016. V. 36. P. 605.

[14] Dedov I.I., Shestakova M.V., Mayorov A.Y. // Diabetes Mellitus. 2019. V. 22. N 1S. P. 1. doi 10.14341/DM221S1

[15] Colwell J.A. Diabetes - Hot Topics. New York: Hanley \& Belfus, 2003. 255 р.; Колуэлл Дж.А. Сахарный диабет: новое в лечении и профилактике. М.: Бином. Лаб. знаний, 2007. $288 \mathrm{c}$

[16] Watkins P.J. // J. Breath Res. 2017. V. 11. P. 024002. doi 10.1088/1752-7163/aa66d3

[17] Ruzsanyi V., Kalapos M. Peter, Breath acetone as a potential marker in clinical practice // J. Breath Res. 11 (2017) 024002. doi 10.1088/1752-7163/aa66d3.

[18] Turner C., Walton C., Hoashi S., Evans M. // J. Breath Res. 2009. V. 3. N 4. P. 046004. doi 10.1088/17527155/3/4/046004

[19] Kistenev Y.V., Borisov A.V., Kuzmin D.A., Bulanova A.A., Boyko A.A., Kostyukova N.Y., Karapuzikov A.A. // Proc. SPIE. 2016. V. 9707. P. 97070M. doi 10.1117/12.2214645

[20] Stepanov E.V., Glushko A.N., Kasoev S.G., Koval A.V., Lapshin D.A. // Quant. Electron. 2011. V. 41. N 12. P. 1124. doi 10.1070/QE2011v041n12ABEH014698

[21] Stepanov E.V., Kasoev S.G. // Opt. Spectrosc. 2019. V. 126. N 6. P. 736. doi 10.1134/S0030400X19060249

[22] Petrus M., Bratu A.-M., Popa C. // Rev. Roum. Chim. 2016. V. 61. N 2. P. 89.

[23] Petrus M., Bratu A.-M., Popa C. // Opt. and Quant. Electron. 2017. V. 49. N 1. doi 10.1007/s11082-016-0837-y

[24] Khodos V.V., Ryndyk D.A., Vaks V.L. // Eur. Phys. J. Appl. Phys. 2004. V. 25. P. 203. doi 10.1051/epjap:2004008

[25] Smolyanskaya O.A., Chernomyrdin N.V., Konovko A.A., Zaitsev K.I., Ozheredov I.A., Cherkasova O.P., Nazarov M.M., Guillet J.-P., Kozlov S.A., Kistenev Y.V., Coutaz J.-L., Mounaix P., Vaks V.L., Son J.-H., Cheon C., Wallace V.P., Feldman Y., Popov I.B., Yaroslavsky A.N., Shkurinov A.P., Tuchin V.V. // Progress in Quant. Electron. 2018. V. 62. P. 1. doi 10.1016/j.pquantelec.2018.10.001
[26] Vaks V.L., Domracheva E.G., Pripolzin S.I., Chernyaeva M.B. // EPJ Web Conf. 2018. V. 195. P. 10014. doi 10.1051/epjconf/201819510014

[27] Demidova T.Yu., Selivanova A.V., Ametov A.S. // Terapevticheskii Arkhiv. 2006. V. 78. N 11. P. 64

[28] Сивухин Д.В. Общий курс физики: Термодинамика и молекулярная физика. / Учебное пособие. М.: Физматлит, 2006. 544 c.

[29] Jolliffe I.T. Principal Component Analysis. N.Y:: Springer, 2002. 405 p.

[30] Scholz M., Fraunholz M., Selbig J. // Principal Manifolds for Data Visualization and Dimension Reduction. / Ed. by Gorban A.N., Kégl B., Wunsch D.C., Zinovyev A.Y. Lecture Notes in Computational Science and Enginee, V. 58. 2008. Berlin, Heidelberg: Springer. P. 44.

[31] Vaks V.L., Domracheva E.G., Sobakinskaya E.A., Chernyaeva M.B. // Phys. Usp. 2014. V. 57. N 7. P. 684. doi 10.3367/UFNe.0184.201407d.0739

[32] Exter M., Fattinger Ch., Grischkowsky D. // Opt. Lett. 1989. V. 14. N 20. P. 1128. doi 10.1364/OL.14.001128

[33] Peale R.E., Muravjov A.V., Fredricksen C.J., Boreman G.D., Saxena H., Braunstein G., Vaks V.L., Maslovsky A.V., Nikifirov S.D. // International J. High Speed Electronics and Systems. 2008. V. 18. N 3. P. 627. doi $10.1142 / \mathrm{S} 012915640800562 \mathrm{X}$ 